\title{
External Quality Assessments of CD31 Immunoassays - the NordiQC experience
}

\author{
Mogens Vyberg, 1, 3, a, @ Søren Nielsen, 1 Michael Bzorek, 2 Rasmus Røge, 1, 3 \\ @ corresponding author, \& equal contributor \\ Vascular Cell. 2020; 12(1):4 | ๔ Mogens Vyberg, Søren Nielsen, Michael Bzorek, Rasmus Røge \\ Received: 19 May 2020 | Accepted: 29 May 2020 | Published: 30 July 2020 \\ Vascular Cell ISSN: 2045-824X \\ DOI: https://doi.org/10.24238/13221-12-1-193
}

Author information
1. Institute of Pathology - Aalborg University Hospital; Aalborg, 9000, Denmark
2. Department of Surgical Pathology - Zealand University Hospital; Roskilde, 4000, Denmark
3. Department of Clinical Medicine - Aalborg University; Aalborg, 9220, Denmark

[a] mov@dcm.aau.dk

\begin{abstract}
Nordic Immunohistochemical Quality Control (NordiQC) performs proficiency testing for about 600 pathology laboratories in more than 50 countries. All general results are published on http://www. nordiqc.org. Over-all, about $30 \%$ of the staining results on circulated slides from tissue micro arrays have been assessed as insufficient for diagnostic use by an expert group. This paper describes the results from the two latest NordiQC runs for CD31. Out of 476 stains submitted, $30.5 \%$ were considered insufficient, mostly due to too weak or false negative staining reactions. The best results were obtained by use of mouse monoclonal antibody JC70A with an optimized protocol based on efficient heat induced epitope retrieval and a three-step polymer/multimer conjugate as visualization system. The mouse monoclonal antibody $1 \mathrm{~A} 10$ gave unsatisfactory results in almost all cases.
\end{abstract}

\section{Keywords}

CD31 - Immunohistochemistry - External quality assurance - NordiQC

\section{Introduction}

CD31 is a transmembrane glycoprotein, about 130 $\mathrm{kDa}$, also designated platelet-endothelium cell adhesion molecule (PECAM-1), belonging to the immunoglobulin super family and encoded by the PECAM-1 gene on chromosome 17 [1]. CD31 expression is restricted to endothelial cells (ECS), platelets and immune cells, suggesting that CD31 is specifically involved in the immune responses at the vascular wall [2]. Thus, during inflammation CD31 plays a major role in the adhesion cascade between endothelial cells and inflammatory cells, facilitating leucocyte migration, particularly being an important component in the regulation of neutrophil transendothelial migration [3]. CD31 also plays a role in thrombosis and angiogenesis.

Immunohistochemically, CD31 is usually strongly expressed in both blood vessel and lymphatic vessel ECs, in a distinct membranous pattern as it makes up a large portion of the intercellular junctions. In liver, the expression in ECs is lower
[4] but increases with capillarization of the liver sinusoids in chronic liver diseases [5]. CD31 is varyingly expressed in myeloid progenitor cells, megakaryocytes, platelets, and neutrophil granulocytes as well as in subpopulations of B- and T-lymphocytes. Typically, mantle zone B-cells have a low expression of CD31, while plasma cells are high expressors. Among neoplasms, CD31 is expressed in the vast majority of vascular lesions, such as haemangioma, angiofibroma, and angiosarcoma, and in most cases of Kaposi sarcoma and epithelioid hemangioendothelioma [6]. In some tumours such as littoral cell angioma, identification of CD31 may be mandatory for a correct diagnosis. CD31 may also be expressed in a minority of haematolymphoid neoplasms like histiocytosis, juvenile xanthogranuloma, B- and T-cell lymphomas, plasmacytoma, and myeloid leukaemia. Among carcinomas and mesotheliomas, also a minority of cases has been reported to express CD31 [7]. CD31 assays are mostly applied in order to identify vascular ECs in various lesions 
(e.g., to visualize metastatic spread in lymphatic and blood vessels), assess angiogenesis (vascular density) in neoplasms and verify endothelial neoplasms. For these purposes, CD31 is often used in panels, which also may include other endothelial markers like ERG, CD34, Fli-1 and Podoplanin (D2-40)[8].

In the Nordic immunohistochemical Quality Control (NordiQC) external quality assessment (EQA) program, CD31 IHC assays have been assessed five

\section{Materials and Methods}

Tissue microarrays (TMAs) for the CD31 tests were constructed for each run comprising tissues from appendix, tonsil, liver, angiosarcoma, and (in run 46) colon adenocarcinoma. The included tissues were all fixed in $10 \%$ neutral buffered formalin for 24-48 hours. Criteria for assessing CD31 staining as optimal included:

- A strong and distinct, predominantly membranous staining reaction of virtually all normal ECs and plasma cells in appendix and tonsil

- An at least weak to moderate, distinct membranous staining reaction of activated $B$ - and T-cells, in particular mantle zone B-cells, in the tonsil and intraepithelial T-cells in the appendix

- An at least weak to moderate staining reaction of the majority of the hepatic sinusoidal ECs

- An at least moderate, predominantly membranous staining reaction of the vast majority of neoplastic cells in the angiosarcoma

\section{Results}

In the two runs (38/2013 and 46/2016), a total of 476 slides stained for CD31 were assessed (Table 1 ). The most commonly used antibody (Ab) was mouse monoclonal $(\mathrm{mm})$ Ab clone JC70A applied in 257 cases, of which $73.9 \%$ provided a sufficient result (optimal or good). Optimal staining results times during 2004-2016. A large proportion of submitted assays has been assessed as insufficient $(24-48 \%)$. The aim of the present paper is to give an overview of the CD31 EQA results in the two latest assessments, run 38 (2013) and run 46 (2016). Detailed assessment reports from all runs are available on the NordiQC homepage (http://www. nordiqc.org).

- No staining reaction of epithelial cells.

Sections from different levels of the TMAs were stained for CD31 in the NordiQC reference laboratory (Lab) using mmAb clone JC70A to ensure an appropriate and constant expression of CD31 throughout the slides to be circulated. Participants submitted their CD31 IHC staining protocols on the NordiQC homepage and received a pair of slides cut from the test TMAs. The participants performed the IHC assays for CD31 according to their submitted protocol and returned one of the slides to NordiQC. An expert assessor panel consisting of experienced pathologists and technicians performed the assessment. The assessor panel performed an anonymized consensus assessment by evaluating each slide on a microscope linked to a projector. Each assay was marked as "optimal," "good," "borderline," or "poor" based on the technical quality and concordance to the staining pattern as outlined above. Optimal and good are considered sufficient, while borderline and poor are insufficient for diagnostic use.

could be obtained on all main platforms (Dako, Leica and Ventana). Among the Lab developed tests (LDTs), the highest proportion of optimal results was obtained by efficient heat induced epitope retrieval (HIER) in an alkaline buffer, TRS low pH (Dako) or DIVA pH 6 (Biocare), in combination with an appropriate calibration of the primary $A b$ and use of a sensitive 3-step detection system.

\section{Table 1}

\section{Pooled staining results related to antibodies in the two latest runs assessed by NordiQC.}

\begin{tabular}{|l|l|l|l|l} 
Clone and format & No. of slides & Optimal (\%) & Good (\%) & Insufficient (\%) \\
\hline JC70A Conc & 257 & 43.6 & 30.3 & 26.1 \\
\hline JC70A RTU Dako & 100 & 57.0 & 35.0 & 8.0 \\
\hline JC70A RTU Ventana & 82 & $30.5^{*}$ & 20.7 & 48.8 \\
\hline 1A10 RTU Leica & 10 & 0.0 & 0.0 & 100.0 \\
\hline 1A10 Conc & 9 & 0.0 & 11.1 & 88.9 \\
\hline Other & 18 & 16.7 & 16.7 & 66.7 \\
\hline Total & 476 & 41.4 & 28.2 & 30.5 \\
\hline
\end{tabular}

*Optimal results could only be obtained by modifying the vendor protocol recommendations.

Conc: Concentrate, RTU: Ready to use 
The mmAb clone JC70A in a ready to use (RTU) product from six vendors was used in 189 cases, of which $73.0 \%$ provided a sufficient result. The RTU products from Dako used in 100 cases gave sufficient results in $92.0 \%$, of which $57 \%$ were optimal. An optimal result could both be obtained by the Dako recommended protocol settings and by Lab modified settings. The RTU product from Ventana used in 82 cases gave sufficient results in $51.2 \%$ and only $30.5 \%$ being optimal. An optimal result with the Ventana product was typically based on a Lab modified protocol using a 3-step detection system as OptiView or UltraView + amplification. In contrast, adherence to the vendor recommended protocol settings, which includes the 2-step visualization system Ultraview, could not provide any optimal staining reaction.

Only few participants used other antibodies than mmAb clone JC70A. The mmAb clone $1 \mathrm{Al0}$ was applied in 20 cases either as a concentrate (Conc) or an RTU product. However, none were optimal, only one was good, and 19 (95\%) were insufficient. Nine slides were stained for CD31 using one of the clones EP3095, UC-CD31, BS50 or EP78 as a Conc or BC2 as an RTU product. None of these provided an optimal result and only BC2 gave good results (both of two stains).

The prevalent feature of insufficient staining results was a "too weak" or "false negative" staining reaction in cells and structures expected to be demonstrated (Figure 1 ). These patterns were observed in $98 \%$ of the insufficient results. Virtually all Labs were able to demonstrate CD31 in high level antigen expressing structures such as ECs of the large vessels in the appendix and portal tracts of the liver, whereas demonstration of CD31 in low level expressing structures as hepatic sinusoidal ECs and activated B-cells in the mantle zones of the tonsil were more challenging. 


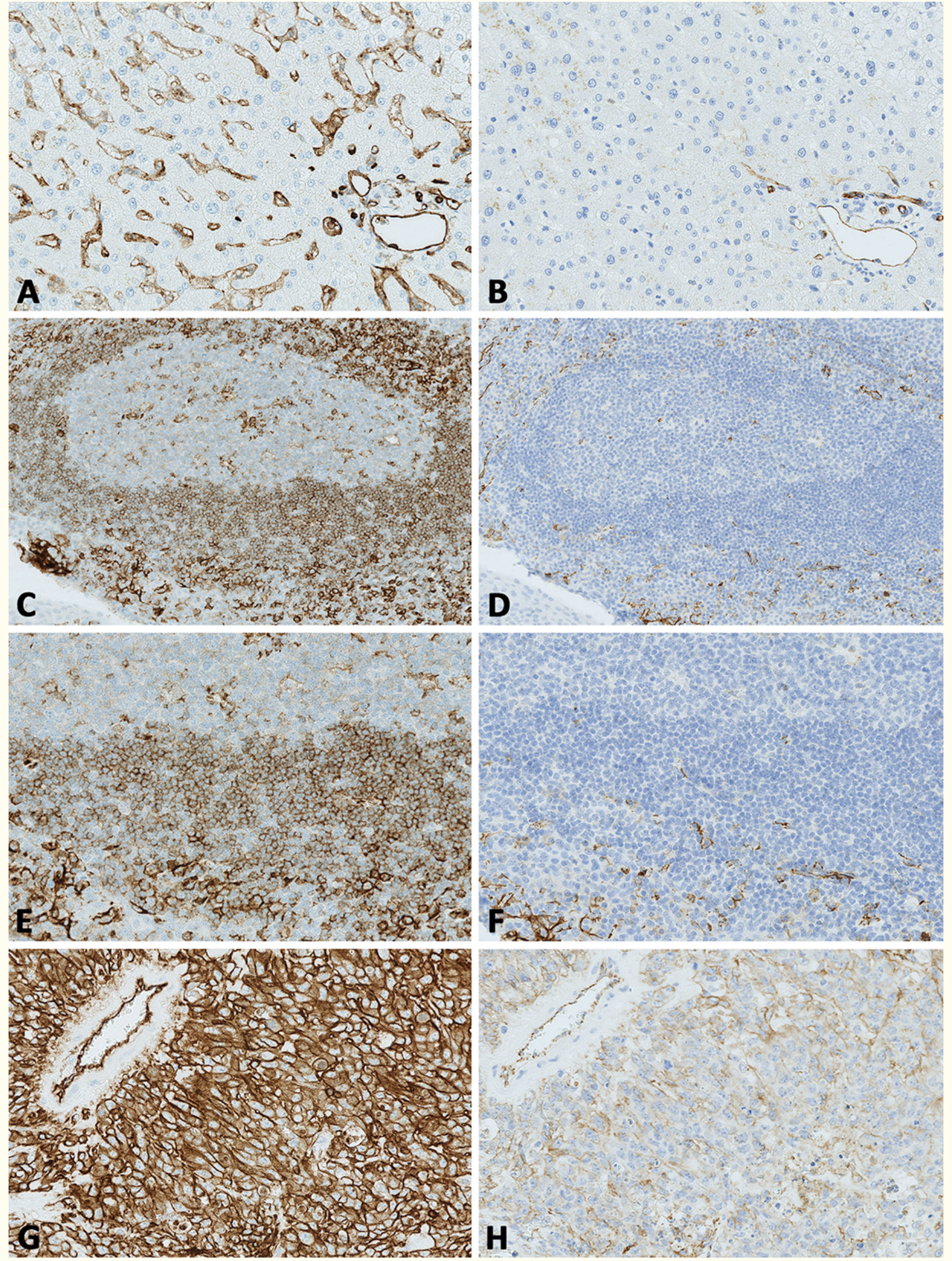

Figure 1: Optimal and insufficient staining results for CD31 as assessed by NordiQC. 


\section{Figure 1: Optimal and insufficient staining results for CD31 as assessed by NordiQC.}

A. Optimal CD31 staining of the liver using the mmAb clone JC70A as a concentrate, HIER in an alkaline buffer and a 3-step multimer based detection system. Virtually all the hepatic sinusoidal endothelial cells show a weak to moderate, predominantly membranous staining reaction.

B. Same field as in A. Insufficient staining for CD31 of the liver using the mmAb clone JC70A in a protocol with a too low sensitivity. Endothelial cells lining large vessels are demonstrated, while the sinusoidal endothelial cells are false negative.

C. Optimal CD31 staining of the tonsil using the mmAb clone JC70A in an RTU format (GA610, Dako), with HIER in TRS High pH 9 for 30 min., a 3-step polymer-based detection kit, performed on the Dako Omnis stainer (vendor recommended protocol settings). Even at low magnification a distinct positive staining reaction of mantle zone B-cells, plasma cells and endothelial cells can be identified.

D. Same field as in C. Insufficient CD31 staining of the tonsil using the mmAb clone JC70A in an RTU format (IR610, Dako) but with laboratory modified protocol settings reducing HIER time and changing detection kit. The endothelial cells are weakly stained, the lymphocytes are false negative. E. Higher magnification of the tonsil in C. A moderate and distinct membranous staining reaction is seen in the majority of mantle zone B-cells, while plasma cells and endothelial cells show an intense staining reaction.

F. Same field as in E, same protocol as in D. Insufficient CD31 staining of the tonsil. Only endothelial cells and plasma cells are demonstrated, while mantle zone B-cells are negative.

G. Optimal CD31 staining of the angiosarcoma using the same protocol as in C and E. Virtually all neoplastic cells show a distinct, predominantly membranous staining reaction.

H. Same field as in G, same antibody and protocol as in D and F. Insufficient CD31 staining of the angiosarcoma. The intensity and proportion of neoplastic cells demonstrated is significantly reduced compared to G. CD31 positive normal endothelial cells are demonstrated (top left) emphasizing that these cells cannot reliably be used as internal positive tissue control.

Foot note: All photos in the multi-panel are obtained by Hamamatsu Nano Zoomer 2.0-RS slide scanner at $20 X(A-B, E-H)$ or $4 X(C-D)$ magnification.

\section{Discussion and Conclusion}

In the NordiQC EQA, about $30 \%$ of all stains in the general module have been assessed as insufficient for diagnostic use [9] ]. About $90 \%$ of insufficient results are characterized by a "too weak" or "false negative" reaction, particularly in cells with a low epitope expression. In most cases, the major causes can be identified as less successful (poor and less robust) antibodies, poorly calibrated RTU products, stainer platform-dependent antibodies, insufficiently calibrated antibody dilutions, insufficient/erroneous epitope retrieval, less sensitive visualization systems, and others (e.g., retrieval induced impaired morphology, drying out phenomena, stainer platform-dependent protocol issues, platforms causing uneven staining reactions).

In the current EQA of CD31 assays, the less successful mmAb clone $1 A 10$ and low sensitivity protocols were prevailing causes of insufficient staining results. Also a significant cause may be the lack of awareness of the immunohistochemical critical assay performance controls (iCAPCs) as it is essential to demonstrate an appropriate and successful level of the analytical sensitivity in the assay for CD31. Liver, tonsil and appendix combined are appropriate as positive and negative control tissues for CD3. In tonsil virtually all plasma cells and ECs lining large vessels should show a moderate to strong staining reaction, whereas the vast majority of mantle zone B-cells must show an at least weak to moderate staining reaction. The positive staining reaction of mantle zone $B$ cells with low-level CD31 expression serves as the ICAPC for the low limit of detection of CD31. In liver, the hepatic sinusoidal ECs are also suitable as iCAPC for CD31, due to low-level CD31 expression. No staining reaction should be seen in e.g., hepatocytes, appendiceal columnar epithelial cells and squamous epithelial cells in tonsil, all serving as negative tissue controls.

Also in this context, it has to be emphasized that these tissue controls should be used together as external on-slide control to confirm the IHC test 
reproducibility. Batch controls and internal controls (ECs in general) for CD31 cannot stand alone as in most instances they do not accurately monitor and assure the IHC analytical test performance [10].

In conclusion, the key points for CD31 immunoassays are as follows:

- Select a sensitive Ab clone, e.g., mmAb JC70A as Conc in an LDT or in a Dako/Agilent RTU format

\section{Original submitted files for images}

Below are the links to the authors' original submitted files for images.
- Do not use an insensitive clone like mmAb clone $1 \mathrm{~A} 10$

- Use an optimized protocol based on HIER and a three-step polymer/multimer conjugate

- Use appropriate on-slide iCAPCs for CD31 (Liver, tonsil and appendix).

Original image file for Figure 1

Click here to view.

\section{References}

1. Lertkiatmongkol P, Paddock C, Newman DK, Zhu J, 6. Naeem N, Mushtaq S, Akhter N, Hussain M, Thomas MJ, Newman PJ. The Role of Sialylated Glycans in Human Platelet Endothelial Cell Adhesion Molecule 1 (PECAM-1)-mediated Trans Homophilic Interactions and Endothelial Cell Barrier Function. J Biol Chem. 2016 Dec 9;291(50):26216-26225. doi:10.1074/ jbc.M116.756502

2. Marelli-Berg FM, Clement M, Mauro C, Caligiuri G. An immunologist's guide to CD31 function in Tcells. J Cell Sci. 2013 Jun 1;125(Pt 11):2343-52. doi:10.1242/jcs. 124099

3. O'Brien CD, Lim P, Sun J, Albelda SM.

PECAM-1-dependent neutrophil transmigration is independent of monolayer PECAM-1 signaling or localization. Blood. $2003 \mathrm{Apr}$ 1;101(7):2816-25. doi:10.1182/

blood-2002-08-2396

4. Lalor PF, Edwards S, McNab G, Salmi M, Jalkanen S, Adams DH. Vascular adhesion protein-1 mediates adhesion and transmigration of lymphocytes on human hepatic endothelial cells. J Immunol. 2002 Jul 15;169(2):983-92. doi:10.4049/ jimmunol.169.2.983

5. Xu B, Broome U, Uzunel M, Nava S, Ge X, Kumagai-Braesch M, Hultenby K, Christensson B, Ericzon BG, Holgersson J, Sumitran-Holgersson S. Capillarization of hepatic sinusoid by liver endothelial cell-reactive autoantibodies in patients with cirrhosis and chronic hepatitis. Am H Pathol. 2003 Oct;163(4):1275-89. doi:10.1016/ S0002-9440(10)63487-6 Hassan U. Effectiveness of Vascular Markers (Immunohistochemical Stains) in Soft Tissue Sarcomas. J Coll Physicians Surg Pak. 2018 May;28(5):352-356. doi:10.29271/ jcpsp.2018.05.352

7. De Young BR, Frierson HF Jr, Ly MN, Smith D, Swanson PE. CD31 immunoreactivity in carcinomas and mesotheliomas. Am J Clin Pathol. 1998 Sep;110(3):374-7. doi:10.1093/ajcp/ 110.3.374

8. Gill R, O'Donnell RJ, Horvai A.. Utility of immunohistochemistry for endothelial markers in distinguishing epithelioid hemangioendothelioma from carcinoma metastatic to bone. Arch Pathol Lab Med. 2009 Jun;133(6):967-72. doi:10.1043/ 1543-2165-133.6.967

9. Vyberg M, Nielsen S. Proficiency testing in immunohistochemistry--experiences from Nordic Immunohistochemical Quality Control (NordiQC). Virchows Arch. 2016 Jan;468(1):19-29. doi:10.1007/s00428-015-1829-1

10. Torlakovic EE, Nielsen S, Francis G, Garratt J, Gilks B, Goldsmith JD, Hornick JL, Hyjek E, Ibrahim M, Miller K, Petcu E, Swanson PE, Zhou X, Taylor CR, Vyberg M. Standardization of positive controls in diagnostic immunohistochemistry: recommendations from the International Ad Hoc Expert Committee. Appl Immunohistochem Mol Morphol. 2015 Jan;23(1):1-18. doi:10.1097/ PAI.0000000000000163

\section{Copyright \& License}

Statement: Copyright (c) 2020, Mogens Vyberg, Søren Nielsen, Michael Bzorek, Rasmus Røge. Holder: Mogens Vyberg, Søren Nielsen, Michael Bzorek, Rasmus Røge Licensee: Publiverse Online S.R.L.

License: Open Access This article is distributed under the terms of the Creative Commons Attribution 4.0 International License (https://creativecommons.org/licenses/by/4.0/), which permits unrestricted use, distribution, and reproduction in any medium, provided you give appropriate credit to the original 
author(s) and the source, provide a link to the Creative Commons license, and indicate if changes were made. The Creative Commons Public Domain Dedication waiver (https://creativecommons.org/ publicdomain/zero/1.0/) applies to the data made available in this article, unless otherwise stated.

\section{Vascularcell P P PUBLIVERSE $_{\mathrm{O}}$}

The present article has been published in Vascular Cell journal by Publiverse Online S.R.L. 